\title{
TU/e ENHOUEN

\section{On the correlation of inverted flame blow-off limits with the boundary velocity gradient at the flame holder surface}

\section{Citation for published version (APA):}

Shoshyn, Y., \& de Goey, L. P. H. (2015). On the correlation of inverted flame blow-off limits with the boundary velocity gradient at the flame holder surface. Combustion, Explosion and Shock Waves, 51(5), 520-527. https://doi.org/10.1134/S0010508215050020

\section{Document license:}

TAVERNE

DOI:

$10.1134 / \mathrm{S} 0010508215050020$

Document status and date:

Published: 01/01/2015

\section{Document Version:}

Publisher's PDF, also known as Version of Record (includes final page, issue and volume numbers)

\section{Please check the document version of this publication:}

- A submitted manuscript is the version of the article upon submission and before peer-review. There can be important differences between the submitted version and the official published version of record. People interested in the research are advised to contact the author for the final version of the publication, or visit the $\mathrm{DOI}$ to the publisher's website.

- The final author version and the galley proof are versions of the publication after peer review.

- The final published version features the final layout of the paper including the volume, issue and page numbers.

Link to publication

\section{General rights}

Copyright and moral rights for the publications made accessible in the public portal are retained by the authors and/or other copyright owners and it is a condition of accessing publications that users recognise and abide by the legal requirements associated with these rights.

- Users may download and print one copy of any publication from the public portal for the purpose of private study or research.

- You may not further distribute the material or use it for any profit-making activity or commercial gain

- You may freely distribute the URL identifying the publication in the public portal.

If the publication is distributed under the terms of Article 25fa of the Dutch Copyright Act, indicated by the "Taverne" license above, please follow below link for the End User Agreement:

www.tue.nl/taverne

Take down policy

If you believe that this document breaches copyright please contact us at:

openaccess@tue.nl

providing details and we will investigate your claim. 


\title{
On the Correlation of Inverted Flame Blow-Off Limits with the Boundary Velocity Gradient at the Flame Holder Surface
}

\author{
Y. Shoshin ${ }^{a}$ and L. P. H. de Goey ${ }^{a}$
}

UDC 662.612 .32

Published in Fizika Goreniya i Vzryva, Vol. 51, No. 5, pp. 13-21, September-October, 2015. Original article submitted April 16, 2014; revision submitted November 17, 2014.

\begin{abstract}
Conductive heat losses from the base of a lean methane-air inverted flame stabilized behind the trailing edge of a thin rod have been experimentally evaluated. The results favor the view that the heat losses to the flame holder play a crucial role in the inverted flame stabilization and blow-off. Simple estimations have been performed, which indicate that the well-established correlation between the mixture composition and the boundary velocity gradient at the flame holder, usually considered as a proof of the flame stretch theory of blow-off, can be explained without involving the flame stretch concept. The suggested explanation of this correlation is based on the assumption that the heat loss to the flame holder is the main factor that determines the inverted flame blow-off behavior and on the similarity between the mechanisms of energy and momentum diffusion in gases $(\operatorname{Pr} \approx 1)$.
\end{abstract}

Keywords: inverted flame, flame stabilization, blow-off, boundary velocity gradient.

DOI: $10.1134 /$ S0010508215050020

\section{INTRODUCTION}

Premixed inverted flames stabilized behind the trailing edge of a thin rod (wire) or a plate installed lengthwise in a combustible mixture flow are often used for studies of flame stabilization mechanisms. Such flames are symmetric and not affected by the environment at the flame anchoring location, which facilitates the interpretation of experimental results and modeling.

Lewis and Von Elbe [1] performed experiments with wire-stabilized inverted flames of lean natural gas-air mixtures and found that, for thin enough wires, the blow-off data obtained for different mixtures could be correlated by using the relation

$$
K=\frac{d U}{d x} \frac{\eta_{0}}{S_{L}}=g_{b} \frac{\eta_{0}}{S_{L}},
$$

where $g_{b}=\frac{d U}{d x}$ is the boundary velocity gradient, i.e., the radial gradient of the axial component of the velocity $U$ near the holder side surface at blow-off, $\eta_{0}$ is

\footnotetext{
${ }^{a}$ Eindhoven University of Technology, Mechanical Engineering Den Dolech 2, 5600MB Eindhoven, The Netherlands; y.s.shoshin@tue.nl.
}

the thermal thickness, and $S_{L}$ is the laminar burning velocity of the planar zero-stretch adiabatic flame. The values of the criterion $K$ determined in [1] on the basis of experimental data ranged from 1 to 2 for different equivalence ratios and were nearly independent of the wire diameter for sufficiently thin wires $(\lesssim 2 \mathrm{~mm})$.

Later, blow-off measurements for lean and rich inverted methane-air flames stabilized on thin plates installed in the middle of a flat channel were performed by Edmondson and Heap [2]. These authors found that the value of $K$ decreased with decreasing stabilizing plate thickness. For the thinnest plate $(0.3 \mathrm{~mm})$ used in [2], flame blow-off occurred at a near-constant, about unity, value of $K$, which led the authors to the conclusion that the correlation would only improve if thinner plates were used. In their subsequent work [3], Edmondson and Heap measured blow-off limits of lean propane-air, butane-air, and ethylene-air inverted flames stabilized with a $0.3-\mathrm{mm}$ thick plate: $K \approx 0.95$. The burning velocities for adiabatic planar flames of the mixtures tested by Edmondson and Heap [2, 3] ranged from 8 to $60 \mathrm{~cm} / \mathrm{s}$. 
Equation (1) was derived in [1] from the formula for the flame stretch rate in a velocity gradient flow given by Karlovitz et al. in [4] and was assumed to yield a value of the Karlovitz number at the flame base at the blow-off limit. Thus, inverted flame blow-off was attributed by Lewis and Elbe [1] to attaining the critical value of the flame stretch rate at the flame base, which was supposed to be related to the mixture boundary velocity gradient. However, as it was pointed out in [5], the formula for the flame stretch rate was derived in [4] for the condition $U \gg S_{L}$, and extension of that formula to the case with $U=S_{L}$, which takes place at the base of the inverted flame, was not justified. Additional evidence that Eq. (1) is likely to give invalid Karlovitz numbers at the flame base of inverted flames was provided by experimental measurements [6] and numerical predictions [7] performed for lean methane-air inverted flames. In both works [6, 7], the local Karlovitz number at the flame base was found to be about an order of magnitude smaller than the value predicted by Eq. (1). There is also another reason to doubt the "flame stretch" blow-off hypothesis, based on the estimation by Eq. (1). According to the recent developments of the flame stretch theory, weakening of a flame by the positive flame stretch rate is possible only for mixtures with Le $>1[8,9]$, while the correlation by Eq. (1) was observed, in particular, for lean methaneair flames $(\mathrm{Le} \approx 0.96)$.

Thus, neither the estimation of the Karlovitz number by Eq. (1) nor the interpretation of inverted flame blow-off as being due to the attainment of the critical value of the Karlovitz number seem to be justified, and a good correlation of experimental data by the parameter $K$ attaining a value close to unity at blow-off limits remains unexplained. Moreover, to our knowledge, no reasonable evidence has been provided in the literature that this correlation is indeed related to the flame stretch effects, and, at the same time, no alternative explanation of this correlation has been suggested.

Even though local flame extinguishment due to the flame stretch was suggested by Lewis and von Elbe [1] as the reason for inverted flame blow-off, they considered the quenching effect of the flame holder as a key factor determining the stabilization mechanism. Two possible mechanisms for the quenching effect were considered: due to the heat losses to the cold flame holder and due to destruction of chain carriers. Computational studies of cold wall quenching of the methane-air flame performed by Sloane and Schoene [10] demonstrated that radical recombination on the cold surface is of minor importance compared to homogeneous recombination in the cooled gas near the wall. Therefore, the quenching effect may take place only when the local heat losses from the flame to the flame holder are noticeable. The qualitative interpretation of the inverted flame stabilization mechanism due to the quenching effect of the stabilizer is as follows. At the flame base, the flame displacement speed is opposite and equal in the absolute value to the local gas velocity. The flame is stable if a small displacement of the flame toward or away from the flame holder results in non-zero propagation of the flame base, opposite to the direction of the flame displacement, so that the flame moves back to its stable location. Because the flame base is located in an accelerating flow, the last statement assumes that variations of the flame speed relative to the gas should have a larger absolute value than the respective variations in the gas velocity. Modifications of the burning velocity required for flame stability are provided by variations in the heat losses due to changes in the flame stand-off distance during displacements of the flame base. According to this interpretation, quenching of the flame due to the flame stretch is not a necessary condition for the occurrence of flame blow-off. As the mixture flow velocity is increased, the flame stand-off distance increases and the heat losses to the flame holder decrease. At some time instant, variations of the heat losses become too small to be able to stabilize the flame.

Recently, results of detailed numerical simulations of lean methane-air flames stabilized on a perforated plate have been reported in $[11,12]$. According to the results of that works, modifications of the local burning velocity at the flame base during small flame displacements are caused predominantly by varying the heat losses from the flame base to the flame holder, while the flame stretch plays a secondary role. Measurements reported in [13] for a lean inverted methane-air flame stabilized behind the trailing edge of a rod $3.0 \mathrm{~mm}$ in diameter showed that heat fluxes to the flame holder were large enough to affect the local burning velocity noticeably even near the flame blow-off limit. The results of [11-13] support the hypothesis that the "heat loss" of inverted flames is the main stabilization/blow-off mechanism. An experimental evaluation of heat fluxes from the flame to the flame holder for a thinner flame holder is still, however, desirable because a $3.0-\mathrm{mm}$ rod used in [13] is already in the range where the critical boundary velocity gradient becomes dependent on the rod diameter.

In the present work, to additionally verify the "heat loss" flame stabilization/blow-off hypothesis, an experimental evaluation of heat fluxes from the base of an inverted flame to the flame holder has been performed for lean methane-air flames stabilized on a brass rod 1 $\mathrm{mm}$ in diameter. 


\section{EXPERIMENTAL}

The flame was stabilized behind the trailing edge of a brass rod $1 \mathrm{~mm}$ in diameter positioned along the centerline of a 32-cm-long Pyrex tube with a $12.5-\mathrm{mm}$ internal diameter. The selected rod diameter is within the range for which a good correlation on the inverted flame blow-off limits by Eq. (1) was found [1]. Cylinder technical methane of $99.5 \%$ purity and dried compressed air were used for preparing the mixtures. The mixtures were prepared in-line with two Bronkhorst mass flow controllers (MFCs) installed in the methane and air gas lines. After combining the air and methane flows, the mixture flowed through a buffer vessel for damping residual fluctuations of the equivalence ratio caused by small inherent instabilities of MFCs. Because the precision of the used MFCs decreases at small flow rates, gas flows corresponding to more than $50 \%$ of the maximum MFCs readings were used in the experiment. The mixture flow through the tube was controlled by using a needle valve and a ball rotameter. The excess mixture flow was directed to the exhaust system via a bypass. A detailed description of the experimental setup used to produce inverted flames in this work can be found elsewhere [13].

\subsection{Heat Flux Measurements}

The measurements were performed for two lean methane-air mixtures with equivalence ratios $\phi=0.7$ and 0.75 . Examples of stabilized inverted flames are shown in Fig. 1. Figure 2 shows a schematic diagram of the diagnostic setup used to evaluate heat fluxes. The rod side surface temperatures were measured by a Thermacam FLIR PM 575 thermo-imaging IR camera operating at the wavelength range of $7.5-13 \mu \mathrm{m}$, equipped with a close-up lens. The trailing edge of the rod was located $\approx 10 \mathrm{~mm}$ above the edge of the tube to provide optical access to the side surface of the end segment of the rod. The half of the rod side surface facing the camera was coated with a layer of soot to increase the surface emissivity. The value used for the emissivity of the soot layer within the camera settings was 0.95 , as recommended by the camera manufacturer. The temperature profiles along the rod were obtained by averaging the measured temperatures over complete horizontal pixel lines. From these profiles, the temperature gradient near the rod trailing edge was then determined.

Figure 3 shows an example of the measured rod surface temperature profile along the rod. As seen in Fig. 3, the temperature profile is not linear within a short segment $(\delta \approx 1 \mathrm{~mm})$ near the rod trailing edge. This deviation from linearity could be caused by the fact that the heat transfer from the flame to the flame holder does not

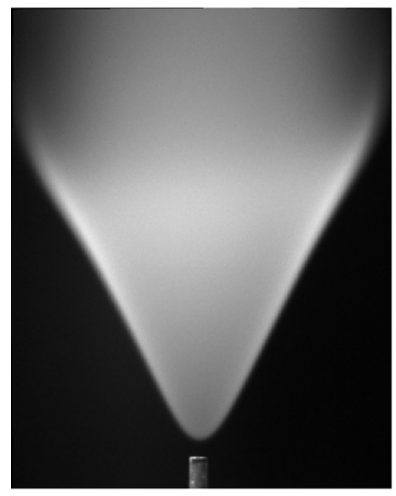

$\phi=0.70$

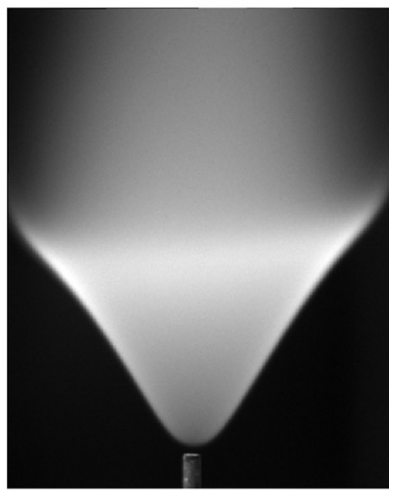

$\phi=0.75$
Fig. 1. Inverted methane-air flames stabilized on the end segment of the rod (the mixture velocity is $U=$ $120 \mathrm{~cm} / \mathrm{s})$.

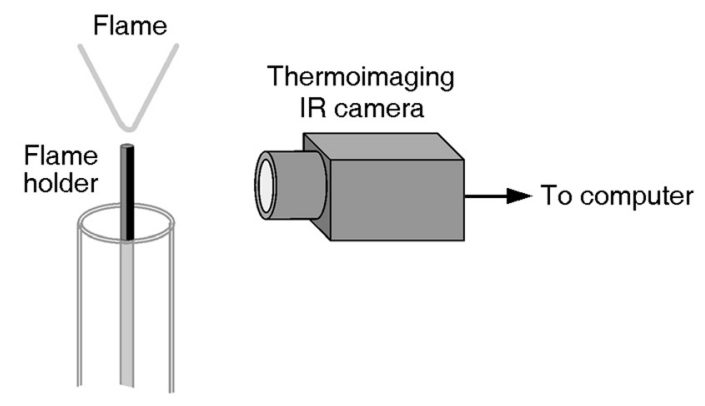

Fig. 2. Schematic diagram of the experimental setup.

only take place through the rod end face plane, but also through the side surface near the rod edge. Therefore, this segment was disregarded, and near-linear temperature profiles along the $\approx 5.5$ - $\mathrm{mm}$ distance below this segment were used for the heat flux evaluation. The heat fluxes were evaluated by the measured temperature profiles by using known values of the rod diameter and of the thermal conductivity of the rod material. The temperature variations within every cross-section of the rod were assumed to be negligible, which was assured by the large ratio of the rod thermal conductivity to the thermal conductivity of the gas mixture. Linear fitting of the measured temperature profiles was used to determine the temperature gradients, which was found to be advantageous compared to fitting by polynomials of higher order. Higher-order polynomials yield more significant scattering in the evaluated temperature gradients, while the average heat fluxes were nearly the same as in the case of linear fitting. The standard devi- 


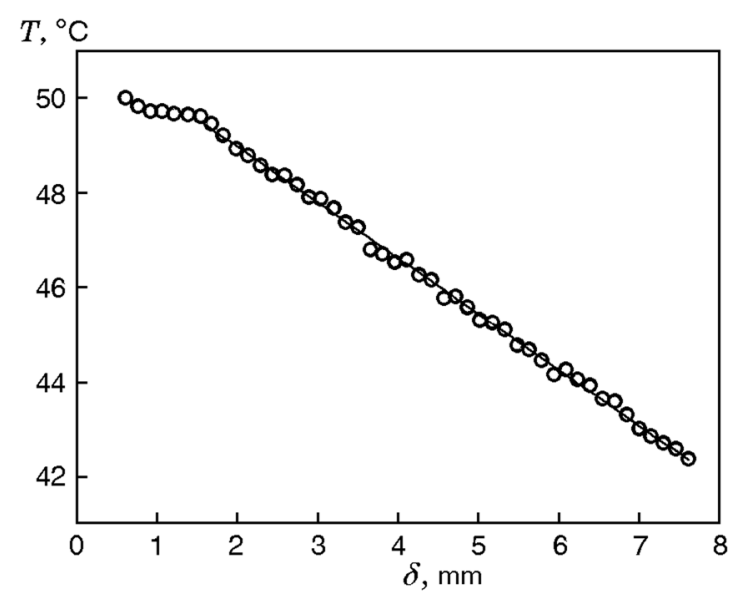

Fig. 3. Measured temperature profile along the rod surface and its fitting ( $\phi=0.75$ and $U=170 \mathrm{~cm} / \mathrm{s})$.

ation of the slopes of the fitted lines did not exceed $3 \%$ of their values in all measurements.

Figure 4a shows the experimentally evaluated heat losses $\dot{q}_{\text {loss }}$ from the flame to the rod. Figure $4 \mathrm{~b}$ shows the heat fluxes normalized by the rate of heat release of a planar methane-air flame of the corresponding mixture, integrated over the front segment equal to the rod cross-sectional area $A: \dot{Q}=\dot{q}_{\text {loss }} / q_{\mathrm{CH}_{4}} Y_{\mathrm{CH}_{4}} S_{b} A$, where $q_{\mathrm{CH}_{4}}$ is the heat of combustion of methane (per unit volume), $Y_{\mathrm{CH}_{4}}$ is the methane volume fraction in the fresh mixture, and $S_{b}$ is the burning velocity of the planar flame for the corresponding mixture. The normalized heat fluxes were determined by using the experimental burning velocities from [14] and the heats of combustion for the corresponding mixtures.

\subsection{Flame Stand-Off Distance Measurements}

The qualitative behavior of the flame stand-off distance versus the mixture inflow velocity was investigated on the basis of direct flame images recorded with a Pike F-032 CCD camera. The stand-off distances $(\Delta)$ were determined by the vertical profiles of the image density $(I)$, as illustrated in Fig. 5. The image density profiles did not have maxima in the flame base region. At the same time, two characteristic branches were clearly distinguishable in the image density profiles: a steep branch, which could be identified as the flame front, and a shallow slope branch. These two branches were interpolated by straight lines, and the distance at which the image intensity reached half of the value corresponding to the intersection of the interpolating lines was taken as the flame base location. The flame stand-off distances versus the mixture velocity obtained in this way are shown in Fig. 6. The mixture
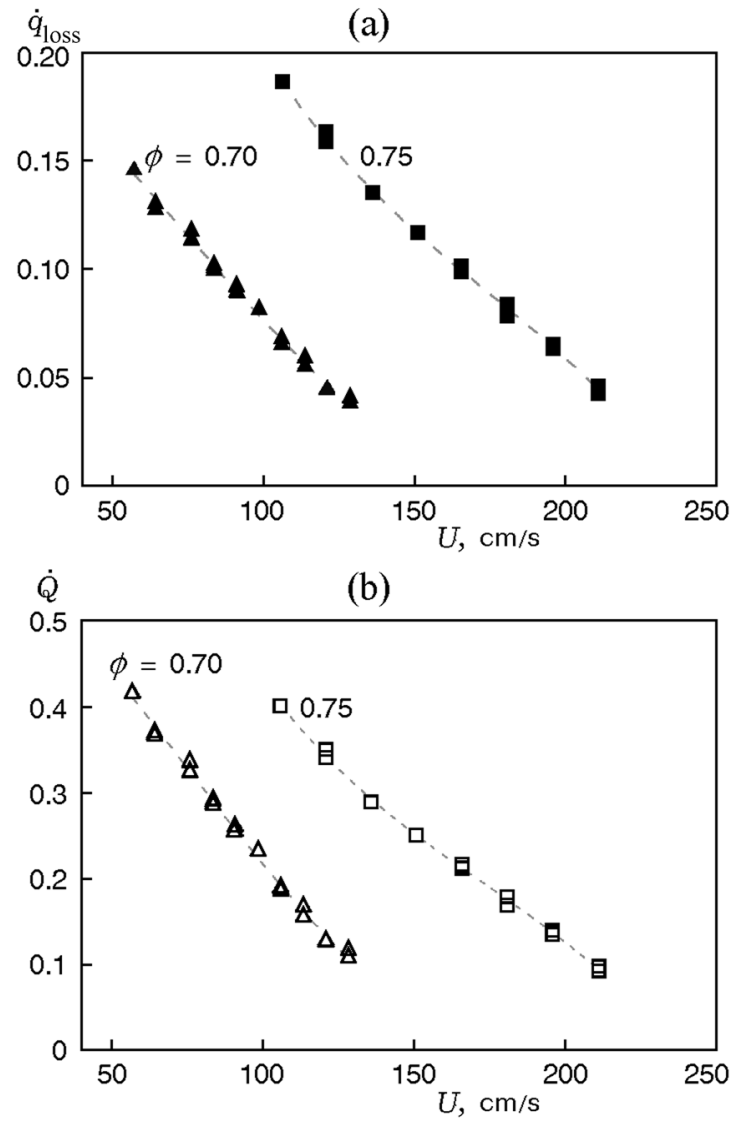

Fig. 4. Experimentally measured heat fluxes to the flame holder (a) and these heat fluxes normalized by the rate of heat release in the flame front with the corresponding area (b) versus the velocity of the mixture.

velocity was determined by dividing the total mixture flow rate by the cross-sectional area of the tube.

Near the blow-off limit, the flame stand-off distance begins to fluctuate in time. The vertical lines in Fig. 6 show the corresponding ranges of the flame stand-off distance fluctuations at near-blow-off mixture velocities. It is seen in Fig. 6 that the stand-off distance grows rapidly as the blow-off limit is approached. The onset of this rapid growth begins when the mixture velocity reaches about $80 \%$ of the critical blow-off value. Near the blow-off limits, the flame can be stabilized at stand-off distances significantly exceeding the flame thermal thickness. At the same time, the heat fluxes to the rod remain noticeable even at near-blow-off mixture velocities. This can be explained by the presence of a vortex between the flame base and the rod trailing edge: the heat transfer between the flame base and the rod edge is intensified by convective transport in the vortex. Numerical simulations [12] for a flame holder 


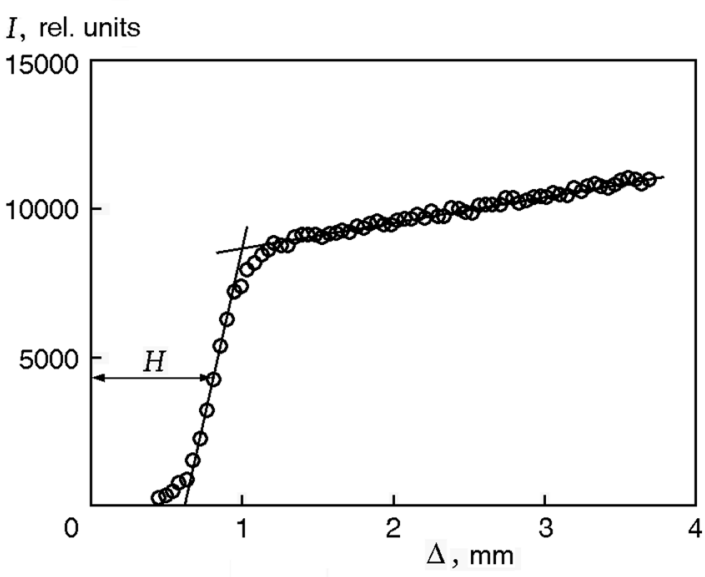

Fig. 5. Image density profiles illustrating the method of determining the distance $H$ from the flame base to the rod end face.

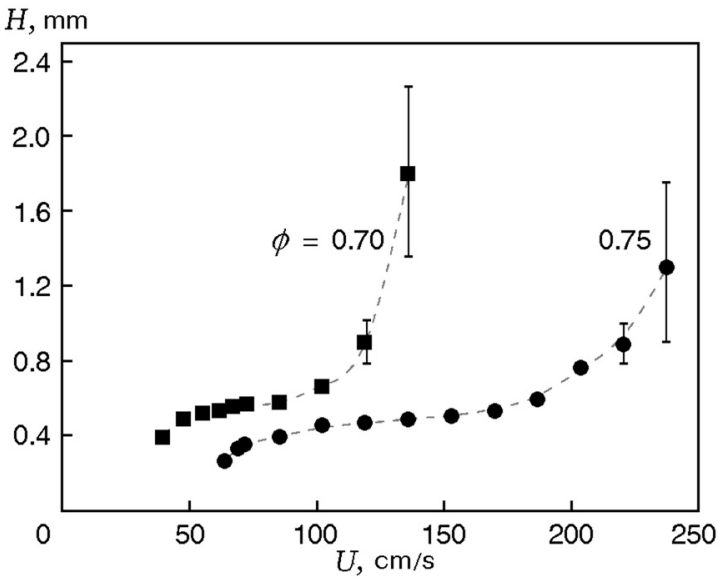

Fig. 6. Distance between the flame base and the rod end face versus the mixture velocity: for mixture velocities close to the flame blow-off limit, timeaveraged values are given.

$1.0 \mathrm{~mm}$ in diameter predict that the vortex size is very small at low mixture velocities and is rapidly growing as the blow-off velocity is approached. Thus, the onset of the rapid increase in the stand-off distance can be identified as the onset of the growth of this vortex.

\section{DISCUSSION OF EXPERIMENTAL RESULTS}

As is observed in Fig. 4, flame blow-off occurs when the conductive heat losses to the rod become relatively small, but still not negligible. The measured heat losses at the blow-off limit constitute $8-10 \%$ of the rate of heat release over the characteristic flame area of a corresponding planar adiabatic flame. This result disagrees with the conclusion of Kawamura et al. [5] and Sung et al. [15] that the heat losses from the flame base to the flame holder can be ignored at the blow-off limit. In [5], the inverted lean methane-air flames were stabilized behind the trailing edge of a metal plate $0.5 \mathrm{~mm}$ thick and $44 \mathrm{~mm}$ wide. The temperatures of the trailing and leading edges of the plate were measured. The conclusion of Kawamura et al. [5] that the role of heat losses to the flame holder is negligible for flame stabilization was based on the small value of the difference $(\approx 10 \mathrm{~K})$ between the two measured temperatures near blow-off. The reliability of this conclusion, however, can be questioned, as quantitative estimations of heat fluxes were not performed in [5]. In [15], the conductive heat losses to the flame holder were estimated for a lean inverted propane-air flame stabilized above the trailing edge of a rod with a diameter of $0.7 \mathrm{~mm}$. Based on the measured difference between the gas temperature at a fixed point and the rod temperature, Sung et al. [15] estimated the heat flux at near-blow-off conditions to be less than $0.3 \%$ of the heat generation rate over the corresponding flame area. The discrepancy with the results of the present work may arise from the fact that convective heat transfer toward the flame holder by the vortex was neglected in the estimations performed in [15]. At the same time, this convective transport may be crucial for stabilizing inverted flames at near-blow-off conditions [12].

As seen in Fig. 4, the heat losses from the flame base to the rod continuously decrease with increasing mixture velocity up to the flame blow-off limit. At the same time, the flame stand-off distance continuously increases with the increase in the mixture velocity (see Fig. 6). These observations fit into the hypothesis that the flame is stabilized due to the quenching effect of the rod. As the mixture velocity increases and the flame recedes from the trailing edge of the rod, the heat flux to the rod decreases. At some time instant, the effect of heat losses on the local burning velocity becomes insufficient to stabilize the flame and the flame is blown off.

It should be emphasized, however, that the fact that the heat losses to the flame holder play a crucial role in inverted flame stabilization does not mean that the flame stretch effects do not affect the flame blowoff limits. As the flame stretch may modify the burning velocity and the flame temperature near the flame base, the flame stretch effects may still influence the critical value of the velocity at which the heat losses to the flame holder become too small to stabilize the flame. 


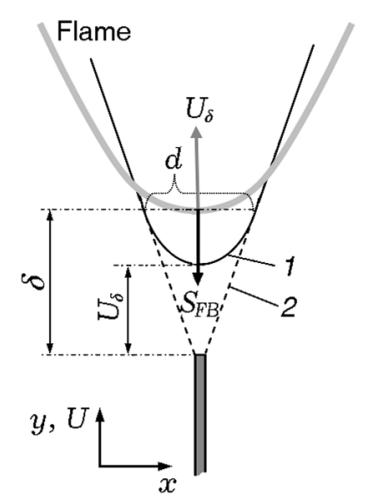

Fig. 7. Inverted flame and basic parameters used for estimation: profiles of $U(x)$ at $y=\delta(1)$ and near the flame holder (2).

The diameter of the rod used in the present work $(1.0 \mathrm{~mm})$ is well within the range for which the correlation of the blow-off limits by Eq. (1) was found to be independent of the rod diameter [1]. At the same time, as discussed in Introduction, no solid proof is found in the literature that this correlation is a result of the flame stretch effects. Taking into account that the present measurements, as well as previously published results [11-13], favor the pure "heat loss" mechanism to be the main flame stabilization/blow-off mechanism, it seems reasonable trying to explain correlation (1) from the standpoint of this mechanism. An attempt of such an explanation, based on order-of-magnitude estimations, is presented in the next section.

\section{POSSIBLE ALTERNATIVE EXPLANATION FOR THE CORRELATION BETWEEN THE INVERTED FLAME BLOW-OFF LIMITS AND BOUNDARY VELOCITY GRADIENTS}

In the experiments described in the previous section, a thin rod was used as a flame holder; nevertheless, the estimations given below are performed for a thin plate flame holder, for simplicity reasons. It is commonly accepted that stabilization mechanisms of rodand plate-stabilized inverted flames are similar, which is also supported by the fact that a good correlation expressed by Eq. (1) was observed for both kinds of flame holders $[1-3]$.

The estimations carried out below are illustrated in Fig. 7. Factors of the order of unity that arise due to averaging procedures or for other reasons are omitted as we are interested only in order-of-magnitude estimates.
The following assumptions are made.

1 . The gas thermal diffusivity, $\alpha$, and the kinematic viscosity $\nu$, are assumed to be constant (equal to their average values) and are close in values $(\operatorname{Pr}=\nu / \alpha \approx 1)$ throughout the flame preheat zone.

2 . The mixture velocity gradient $y$ near the trailing edge of the flame holder is constant along the $x$ axis. This assumption is supported by the experimental fact observed for thin flame holders: correlation (1) for inverted flame stabilization is valid for different initial mixture velocity profiles, but with the same value of the boundary velocity gradient at the flame holder side surface. This is only possible if the flow near the flame holder, where the boundary velocity gradient is nearly constant, affects the flame blow-off limits.

3 . The effect of the flame stretch on the burning velocity is neglected in the estimations. This assumption is justified by numerical predictions of [7, 12], according to which, at varying the mixture inflow velocity, the local burning velocity at the flame base changes mainly because of the variations in the heat losses to the flame holder.

4. It is assumed that the flame holder is so thin that the wake formed above the rod trailing edge is very small, and its size can be neglected compared to the flame diffusion thickness. Thus, we consider the limiting case of a very thin flame holder, for which, hypothetically, the quality of the flame blow-off limit correlation Eq. (1) would be the best $[2,3]$. The local burning velocity at the flame base $S_{\mathrm{FB}}$ at the blow-off limit is assumed to be close in value to the burning velocity of the adiabatic planar flame of a corresponding mixture $S_{\mathrm{FB}} \approx S_{L}$. This assumption is based on the fact that flame blow-off occurs when the heat losses to the flame holder become small.

According to the assumption of a small thickness of the stabilization plate, the mixture velocity profile along the direction normal to the stabilizing plate has a sharp minimum just above the plate trailing edge (see Fig. 7). Due to the gas viscosity, the mixture velocity profile eventually smoothens in some region near the flow centerline downstream as the gas flows past the trailing edge of the plate. The characteristic transversal size of this region at the flame stand-off distance $\delta$ can be estimated by the order of magnitude as

$$
d \sim \sqrt{\nu t_{\delta}}
$$

where $t_{\delta}$ is the characteristic travel time of a gas particle over the flame stand-off distance $\delta$. During the smoothening of the central part of the velocity profile, the gas velocity "averages" over the characteristic size $d$. An order-of-magnitude estimate for the gas axial velocity $y$ at the centerline, at the distance $\delta$ from the trailing 
edge can be obtained by averaging the initial gas velocity profile shown in Fig. 7 over the distance $d$ :

$$
U_{\delta} \approx g_{b} d, \text { i.e., } d \approx U_{\delta} / g_{b} .
$$

The travel time of a gas particle over the distance $\delta$ can be estimated by its average velocity, which changes from 0 at $y=0$ to $U_{\delta}$ at $y=\delta$ :

$$
t_{\delta} \approx \delta / U_{\delta} .
$$

Substituting $d$ and $t_{\delta}$ from Eqs. (3) and (4) into Eq. (2) yields

$$
\frac{U_{\delta}}{g_{b}} \approx \sqrt{\frac{\delta \nu}{U_{\delta}}} .
$$

The estimations Eq. (2)-(4) are physically transparent, though lacking strictness. Therefore, it seems reasonable to verify these estimations on a more faithful base. According to Eq. (5), the $y$ component of the gas velocity $U$ at the centerline past the trailing edge can be expressed as

$$
U \approx \sqrt[3]{g_{b}^{2} \nu y} .
$$

A solution for the gas velocity ( $y$ component) distribution behind an infinitely thin plate of length $l$ in a uniform gas stream was found by Goldstein [16] as a power series, in a fractional power of the distance along the plate from its edge, with numerically calculated coefficients. For the flow centerline, this solution is written as

$$
U=U_{\infty} \sum_{i=0}^{\infty} \alpha_{i}\left(\sqrt[3]{\frac{y}{4 l}}\right)^{1+3 i},
$$

where $U_{\infty}$ is the gas velocity ahead of the plate and $y$ is the distance from the plate trailing edge. Because of the assumption that the boundary velocity gradient is constant at the edge of the plate, which corresponds to a low value of $y / 4 l$, only the first term of the series can be taken for estimation of the axial gas velocity (with the numerical value $\alpha_{0}=1.2262$ found in [16]):

$$
U=1.226 U_{\infty}(y / 4 l)^{1 / 3} .
$$

Based on the Blasius solution [17] for the velocity distribution in the laminar boundary layer employed by Goldstein [16] as a boundary condition at $y=0$, the boundary velocity gradient at the trailing edge of the plate can be related to $U_{\infty}$ as

$$
\left(\frac{\partial U}{\partial x}\right)_{y=0}=g_{b}=0.332 \sqrt{\frac{U_{\infty}^{3}}{\nu l}} .
$$

Equations (8) and (9) yield the equation

$$
U=1.611 \sqrt[3]{g_{b}^{2} \nu y}
$$

which is in agreement with Eq. (6) and confirms the validity of estimations (2)-(5). Because the influence of the heat losses on the local burning velocity near the flame base becomes insignificant at the blow-off limit, the near-blow-off flame stand-off distance $\delta$ should be of the same order of magnitude as the characteristic thermal thickness of the adiabatic planar flame:

$$
\delta \sim \eta_{0} .
$$

Taking into account the assumptions that $\nu \sim \alpha$ and

$$
U_{\delta}=S_{\mathrm{FB}} \sim S_{L},
$$

the value of $\nu / U_{\delta}$ in Eq. (5) can be estimated as

$$
\frac{v}{U_{\delta}} \approx \frac{\alpha}{S_{L}} \approx \eta_{0} .
$$

Substituting Eq. (12), (11), and (13) into Eq. (5), we obtain

$$
\frac{S_{L}}{g_{b}} \approx \eta_{0}, \text { i.e., } g_{b} \frac{\eta_{0}}{S_{L}} \approx 1,
$$

which is the same as Eq. (1) at $K \approx 1$.

Thus, the above-made estimations demonstrate that the correlation of the inverted flame blow-off limits by Eq. (1) with $K \approx 1$ can be explained based on the pure "heat loss" mechanism for flame stabilization. From this standpoint, the flame stretch/preferential diffusion effects, instead of being the reason for the blowoff limit correlation described by Eq. (1), are likely to be the reason for deviations from this correlation. For example, the measurements [2] performed for lean and rich inverted methane-air flames showed that blow-off of rich flames occurred at lower mixture velocities, corresponding to smaller values of $K$. This is consistent with the fact that the flame stretch/preferential diffusion effects in lean and rich methane-air mixtures are opposite: the positive flame stretch at the flame base strengthens lean methane-air flames $(\mathrm{Le}<1)$ and weakens rich methane-air flames (Le $>1)$. In the case where the mixture Lewis number is significantly different from unity, the stretch effects may even qualitatively alter the flame blow-off behavior, like it was found, for example, for lean hydrogen-methane-air flames [13]. In this case, the correlation by Eq. (1) may become invalid.

The above-made estimations were performed for the "idealized" case of a very thin flame holder, for which the vortex above the flame holder can be neglected. Therefore, it can be expected that the quality of the correlation becomes worse with increasing flame holder thickness, which is in agreement with the experimental results presented in $[1,2]$. At the same time, a satisfactory correlation of the blow-off limit prescribed by Eq. (1) was still observed in [1] for experimental conditions at which the wake formed above the flame holder could not be neglected. Neglecting the vortex can, to some extent, be justified by the conclusion drawn by 
Lewis and Von Elbe [18], based on their blow-off measurements performed for wire-stabilized inverted flames: "The vortex seems to be unimportant for the attachment of the flame, because it was found that the critical flow for blow-off did not change when the end of the wire was rounded."

\section{CONCLUSIONS}

The experimental results of this work favor the hypothesis that the heat losses from the flame base to the flame holder play a crucial role in the stabilization/blow-off mechanism of inverted flames. A new explanation of the so-called boundary velocity gradient correlation of blow-off limits of inverted flames is proposed, which is based on the "heat loss" flame stabilization mechanism and does not involve the flame stretch effects. The basis underlying the suggested explanation is related to the similarity between the mechanisms of energy and momentum transfer in gases and the near-unity value of the Prandtl number resulting from this similarity.

The financial support of the Dutch Technology Foundation (STW, Project No. 11616) is gratefully acknowledged.

\section{REFERENCES}

1. B. Lewis and G. von Elbe, Combustion, Flames and Explosions of Gases (Academic Press, New York-London, 1961).

2. H. Edmondson and M. P. Heap, "Blowoff of Inverted Flames," Combust. Flame 14 (2), 191-194 (1970).

3. H. Edmondson and M. P. Heap, "The Correlation of Burning Velocity and Blowoff Data by the Flame Stretch Concept," Combust. Flame 15 (2), 179-187 (1970).

4. B. Karlovitz, D. W. Denniston (Jr.), D. H. Knapschaefer, and F. E. Wells, "Studies on Turbulent Flames," Proc. Combust. Inst. 4, 613-620 (1953).

5. T. Kawamura, K. Asato, T. Mazaki, T. Hamaguchi, and H. Kayahara, "Explanation of the Blowoff of Inverted Flames by the Area-Increase Concept," Combust. Flame 35, 109-116 (1979).
6. T. Kawamura, K. Asato, and T. Mazaki, "Reexamination of the Blowoff Mechanism of Premixed FlamesInverted Flames," Combust. Flame 45, 225-233 (1982).

7. R. M. M. Mallens, L. P. H. de Goey, and C. K. Law, "A Numerical Study of the Stabilisation of a Lean Laminar Premixed V-Shaped Flame," Combust. Sci. Technol. 159, 373-389 (2000).

8. C. K. Law, "Dynamics of Stretched Flames," Proc. Combust. Inst. 22 (1), 1381-1402 (1989).

9. L. P. H. de Goey and J. H. M. ten Thije Boonkkamp, "Mass Burning Rate of Premixed Stretched Flames: Integral Analysis Versus Large-Activation-Energy Asymptotics," J. Eng. Math. 62 (1), 67-84 (2008).

10. T. M. Sloane and A. Y. Schoene, "Computational Studies of End-Wall Flame Quenching at Low Pressure: The Effects of Heterogeneous Radical Recombination and Crevices," Combust. Flame 49 (1-3), 109-122 (1970).

11. H. M. Altay, K. S. Kedia, R. L. Speth, and A. F. Ghoniem, "Two-Dimensional Simulations of Steady Perforated-Plate Stabilized Premixed Flames," Combust. Theory Model. 14 (1), 125-154 (2010).

12. K. S. Kedia and A. F. Ghoniem, "Mechanisms of Stabilization and Blowoff of a Premixed Flame Downstream of a Heat-Conducting Perforated Plate," Combust. Flame 159 (3), 1055-1069 (2012).

13. Y. Shoshin, R. J. M. Bastiaans, and L. P. H. de Goey, "Anomalous Blow-off Behavior of Laminar Inverted Flames of Ultra-Lean Hydrogen-Methane-Air Mixtures," Combust. Flame 160 (3), 565-576 (2013).

14. K. J. Bosschaart and L. P. H. de Goey, "The Laminar Burning Velocity of Flames Propagating in Mixtures of Hydrocarbons and Air Measured with the Heat Flux Method," Combust. Flame 136 (3), 261-269 (2004).

15. C. J. Sung, C. K. Law, and A. Umemura, "On Adiabatic Stabilization of Inverted Flames," Proc. Combust. Inst. 24 (1), 205-212 (1992).

16. S. Goldstein, "Concerning Some Solutions of the Boundary Layer Equations in Hydrodynamics," Proc. Cambridge Phil. Soc. 26 (1), 1-30 (1930).

17. H. Blasius, "Grenzschichten in Flüssigkeiten Mit Kleiner Reibung," Z. Math. Phys. Bd 56, 1-37 (1908).

18. B. Lewis and G. von Elbe, "Stability and Structure of Burner Flames," J. Chem. Phys. 11, 75-97 (1943). 